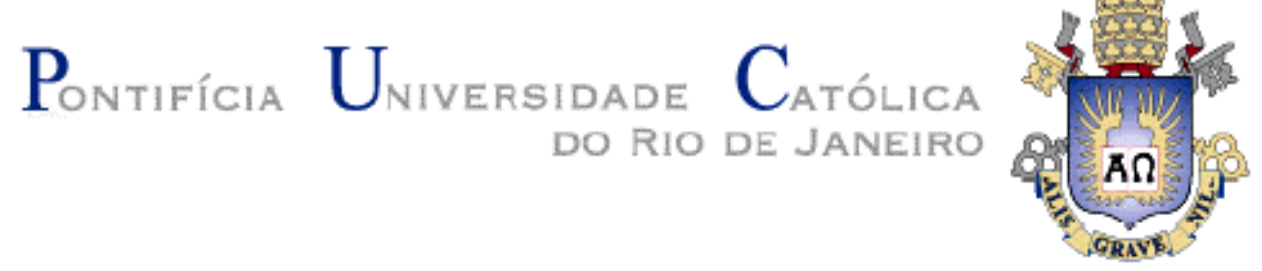

Tatiana Kocerginskis

Redes sociais: a coexistência de classes sociais distintas em um mesmo ambiente digital.

Dissertação de Mestrado Dissertação apresentada ao Programa de Pósgraduação em Administração de Empresas da PUCRio como requisito parcial para obtenção do título de Mestre em Administração de Empresas.

Orientador: Prof. Paulo Cesar Mendonça Motta

Rio de Janeiro

Abril 2012 


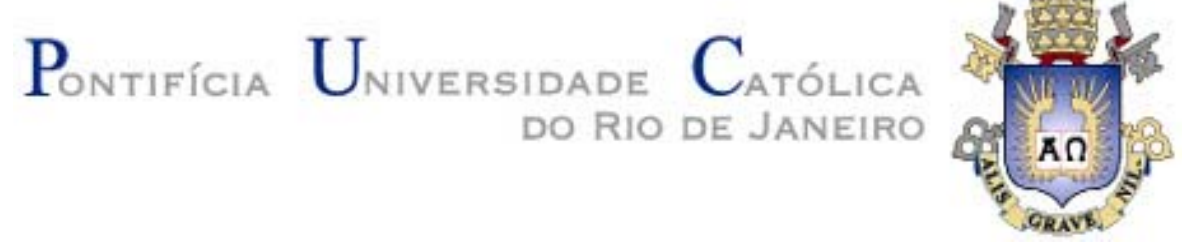

Tatiana Kocerginskis

\title{
Redes sociais: a coexistência de classes sociais distintas em um mesmo ambiente digital
}

\begin{abstract}
Dissertação apresentada como requisito parcial para obtenção do grau de Mestre pelo Programa de Pósgraduação em Administração de Empresas da PUC-Rio. Aprovada pela Comissão Examinadora abaixo assinada.
\end{abstract}

Prof. Paulo Cesar de Mendonça Motta

Orientador

Departamento de Administração - PUC-Rio

Prof. Angeluccia Bernardes Habert Departamento de Comunicação - PUC-Rio

Prof. Manoel Marcondes Machado Neto Faculdade de Administração e Finanças - UERJ

Profa. Mônica Herz

Vice-Decana de Pós-Graduação do CCS

Rio de Janeiro, 16 de abril de 2012 
Todos os direitos reservados. É proibida a reprodução total ou parcial do trabalho sem autorização da universidade, do autor e do orientador.

Tatiana Kocerginskis

Formada na Escola Alemã Corcovado. Graduada em Administração de Empresas pelo IAG/PUC-Rio onde também concluiu o curso de Mestrado em Administração de Empresas com ênfase na área de Marketing.

Ficha Catalográfica

Kocerginskis, Tatiana

Redes sociais: a coexistência de classes sociais distintas em um mesmo ambiente digital / Tatiana Kocerginskis ; orientador: Paulo Cesar Mendonça Motta. - 2012.

72 f. : il. (color.) ; $30 \mathrm{~cm}$

Dissertação (mestrado)-Pontifícia Universidade Católica do Rio de Janeiro, Departamento de Administração, 2012.

Inclui bibliografia

1. Administração - Teses. 2. Rede sociais. 3. Baixa renda. 4. Demarketing. I. Motta, Paulo Cesar Mendonça. II. Pontifícia Universidade Católica do Rio de Janeiro. Departamento de Administração. III. Título.

CDD: 658 


\section{Agradecimentos}

Ao meu orientador, Paulo Cesar Motta, pela sua amizade e por compartilhar seu conhecimento e experiência.

Ao José Roberto Potsch, grande amigo e sempre presente na minha vida acadêmica.

À minha família, que sempre me deu apoio e amor incondicional, sem as quais não estaria apta a concluir esta pesquisa. São elas minha amada irmã, Helena Kocerginskis, e minha tia Sonia Leote, meu pai, Charles David Kocerginskis e sua esposa Maria Ângela Scatena. À Angela um agradecimento especial por reler minha dissertação, corrigindo os erros de português que meus olhos não viam mais.

Aos meus amigos-irmãos, Julio Fleichman, Laura Carvalho, Lucila Campos, a família que eu escolhi, e ao meu namorado Guilherme Netto, que acompanhou de perto a loucura que é trabalhar e dissertar.

Por fim, a todos os meus entrevistados, e aos professores da minha banca, que cederam conhecimento, tempo e boa vontade.

Em memória da minha mãe, Rosa Maria Deffense Leote. 


\section{Resumo}

Kocerginskis, Tatiana; Motta, Paulo Cesar de Mendonça. Redes sociais: a coexistência de classes sociais distintas em um mesmo ambiente digital. Rio de Janeiro, 2012. 72p. Dissertação de Mestrado - Departamento de Administração, Pontifícia Universidade Católica do Rio de Janeiro.

O trabalho visa levantar hipóteses sobre a convivência de diferentes classes sociais em um mesmo ambiente digital. Para isto o trabalho é divido em duas partes, sendo a primeira o referencial teórico, que busca ilustrar conceitos que serão utilizados no modelo. O estudo se inicia com um detalhamento de redes socias, mostrando sua evolução. Depois são aprofundados os seguintes construtos:a) reatância, que mostra que a perda de uma liberdade tem uma influência negativa para o consumidor; b) de demarketing, conceito que indica que algumas vezes é preciso "expulsar” o consumidor indesejado, para manter o desejado e por fim $c$ ) classes sociais, e a forma como as pessoas se relacionam. Após esta etapa foram realizadas entrevistas em profundidade com pessoas que utilizam redes sociais freqüentemente, e que realizaram a migração do Orkut para o Facebook no período em que a baixa renda começou a utilizar a internet e as redes sociais. Ao resumir as idéias principais das entrevistas chegou-se à hipótese de que as pessoas das classes de renda mais altas têm dificuldade em conviver com outras de classes mais baixas, na mesma rede social. O sucesso das redes sociais, em especial o Facebook, estaria condicionado à sua capacidade de aprimorar as ferramentas de privacidade, criando diferentes redes sociais dentro da mesma rede social.

\section{Palavras-chave}

Redes sociais; baixa renda; demarketing. 


\section{Abstract}

Kocerginskis, Tatiana; Motta, Paulo Cesar de Mendonça (Advisor). Social networks: the coexistence of different social classes in the same digital environment. Rio de Janeiro, 2012. 72p. MSc. Dissertation - Departamento de Administração, Pontifícia Universidade Católica do Rio de Janeiro.

The work aims to raise hypotheses about the coexistence of different social classes in the same digital environment. The work is divided into two parts, the first part is the references, which illustrate concepts that are used in the model. This study begins with the explanation of social network, showing their evolution. Then, constructs are defined. They are: reactance, which shows that the loss of freedom has a negative influence for the consumer, demarketing which indicates that it is sometimes necessary to chose "expel" the consumer that you do not want, to maintain the desired one, and finally social classes, and how people relate. After this phase in-depth interviews were carried out with people who frequently use social networks, and that migrated from Orkut to Facebook in the period in which the poor began to use the internet and social networks, in special the Orkut. Once summarize the main ideas of the interviews the result was the hypothesis that people of higher classes have difficulty in beeing in the same social network of the one of the lower class. The success of social networks, especially Facebook, would be conditioned on its ability to enhance the privacy tools, creating different social networks in the same social network.

\section{Keywords}

Social networks; low income; demarketing. 


\section{Sumário}

1. Introdução 12

1.1. Motivação 12

1.2. Relevância do Estudo 13

1.3. Delimitação do Estudo 13

1.4. Considerações 13

1.5. Objetivos 14

2. Referencial Teórico e Revisão de Literatura 15

2.1. Redes Sociais $\quad 15$

2.1.1. Histórico 17

2.1.2. Estatísticas 20

2.1.3. Myspace $\quad 25$

2.1.4. Facebook 26

2.1.5. Orkut 28

2.1.6. A Divisão das Classes Sociais nas Redes Sociais 30

2.1.7. Ruptura 32

2.2. Conceitos Sociais 33

2.2.1. Classes Sociais 33

2.2.2. Construção Social 36

2.2.3. Movimentos Sociais $\quad 37$

2.2.4. Espaço Social $\quad 37$

2.2.5. Capital Social $\quad 39$

2.2.6. Consumo 40

2.3. Teoria da Reatância 41

2.4. Demarketing 42

2.5. Proposição da Pesquisa 43

3. Metodologia 45

3.1. População e Amostra 45

3.2. Coleta de Dados 45

3.2.1. Entrevistas Preliminares 46

3.2.2. Entrevistas em Profundidade 46

3.3. Tratamento dos Dados $\quad 47$

3.4. Limitação do Método 47

3.7. Passos 47 
4. Resultados 49

4.1. Descrição dos Resultados 49

4.2. Construção do Modelo 49

4.2.1. Modelo Inicial $\quad 50$

4.2.2. Pertencimento 50

4.2.3. Movimento 51

4.2.4. Convivência entre Classes Sociais $\quad 52$

4.2.5. Pertencimento 53

4.2.6. Movimento $\quad 56$

4.2.7. Convivência entre Classes Sociais $\quad 57$

4.3. Modelo Final 65

5. Conclusões: Hipóteses 66

5.1. Sugestões e Recomendações para Novas Pesquisas 67

6. Referência Bibliográfica 68 


\section{Lista de figura}

Figura 1: Cronograma das datas de lançamento de muitos sites de rede social

Figura 2: Gay rack $\quad 19$

Figura 3: Catster $\quad 19$

Figura 4: Path 20

Figura 5: Participam de sites de relacionamento, como Orkut (em milhões de usuários)

Figura 6: Números globais de usuários de internet

Figura 7: Top 20 dos principais países em termos de usuários de internet versus população

Figura 8: Comparação de usuários ativos por tempo de utilização e nacionalidade

Figura 9: Myspace

Figura 10: Facebook (Login) 26

Figura 11: Facebook $\quad 27$

Figura 12: As redes sociais no mundo $\quad 27$

Figura 13: Crescimento no número de usuários por país 28

Figura 14: A queda na usabilidade do Orkut 29

Figura 15: Orkut 29

Figura 16: Renda familiar x Utilização de redes sociais 30

Figura 17: Beautiful People Rede Social 32

Figura 18: Duas dimensões do espaço social 38

Figura 19: Três dimensões do espaço social 38

Figura 20: A liberdade e seus efeitos quando a mesma é afetada 42 


\section{Lista de gráficos}

Gráfico 1: Percentual das pessoas que utilizaram a internet, no período de referência dos últimos três meses, na população de 10 anos ou mais de idade, segundo os grupos de anos de estudo. Brasil - 2005/2008

Gráfico 2: Percentual das pessoas que utilizaram a internet, na população de 10 anos ou mais de idade, que utilizou a internet, no periodo de referencia dos últimos tres meses, por finalidade do acesso à internet. Brasil 2005/2008 
"He who loves practice without theory is like the sailor who boards ship without a rudder and compass and never knows where he may cast."

Leonardo Da Vinci 